\title{
ARTIGOS
}




\section{Patrimonio comercial y turismo. Los establecimientos emblemáticos de Barcelona turística}

Retail heritage and tourism. Emblematic shops in Barcelona

Carles Carreras ${ }^{1}$

Lluís Frago ${ }^{2}$

1 Dr. Carles Carreras Verdaguer é titular de Geografia Humana na Universidade de Barcelona e Académico da Real Academia de Boas Letras de Barcelona. Email: ccarreras@ub.edu

2 Dr. Lluís Frago Clols, es especialista en geografía urbana y ejerce de profesor en el Departamento de Geografía y en la Escuela Superior de Comercio y Distribución de la Universidad de Barcelona. Email: Ifrago@ub.edu 


\title{
Resumen
}

En el presente artículo los autores realizan un análisis de las políticas municipales de Barcelona para la protección de establecimientos comerciales que se consideran emblemáticos. En este sentido, en primer lugar, se revisa la dificultad de patrimonializar unas actividades económicas privadas muy dinámicas, para, a continuación estudiar el posible impacto del éxito turístico de la ciudad en la conservación de algunos establecimientos. En tercer lugar, se apunta el fuerte impacto de la evolución de los precios del suelo y de los alquileres en la supervivencia de muchos establecimientos comerciales, para presentar algunas conclusiones de cara a conciliar la tradición y la modernidad en las estructuras comerciales urbanas.

Palabras clave: Barcelona, establecimiento emblemático, patrimonio, turismo.

\begin{abstract}
The authors analyze in this paper the local policies of Barcelona in regard to the conservation of the emblematic shops. First, they study the difficulties to consider heritage the dynamic economic activities, and the impact of the Barcelona's tourist success into the commercial structure. The strong impact of the real estate market into the retail business is also analyzed, especially the rent cost in order to maintain shops open. Some conclusions are presented trying to combine tradition and modernity in the retail activities.
\end{abstract}

Key Words: Barcelona, emblematic shop, heritage, tourism.

\section{INTRODUCCIÓN}

Las complejas interrelaciones entre ciudad y comercio ha sido el tema de estudio de nuestro grupo de trabajo desde 1986, a raíz de la realización de una exhaustiva investigación sobre las áreas de concentración comercial de Barcelona, financiado por la Cámara de Comercio (Carreras, Domingo, Sauer, 1990). Se definía una ciudad de compras unos años antes de los fastos olímpicos y muchos más del éxito turístico de la ciudad, del cual muchos piensan que está muriendo, mayoritariamente quienes lo están matando: los formadores de opinión pública y unos indefinidos "vecinos" pendientes de coherencia. Entre 1997 y 1999 realizamos un inventario de elementos de interés paisajístico para la Agencia del Paisaje Urbano de la ciudad de Barcelona, en el cual, entre otros elementos, se recogieron ya algunos de los llamados establecimientos comerciales emblemáticos; históricos según la definición acuñada por la agencia desde un punto de vista formal arquitectónico (AA.VV., 1999). La agencia, dentro del programa de embellecimiento del espacio público de 
la ciudad montada a caballo de los fastos olímpicos, había organizado la campaña Barcelona ponte guapa! En la que se recogían los establecimientos comerciales de algún valor arquitectónico o de diseño, que tenían en torno a cien años o más y que no habían cambiado su decoración. En la publicación del Atlas comercial de Barcelona, en 2003, se recogió y se valoró dicha campaña (Carreras, 2003). Entre tanto, el fin de la moratoria de la ley de liberalización de los alquileres en España, el famoso decreto Boyer de $1985^{3}$, junto con el paso de los años, treinta años hasta su aplicación definitiva!, y los cambios económicos y sociales generados por la integración a la Unión Europea, en1986, y a la unión monetaria, en 2001, y a la crisis financiera mundial del 2007 iniciaron una oleada de cierre de estos (y muchos otros establecimientos) que ha levantado una enorme polvareda mediática. Dicha polvareda se enmarca, de forma harto incomprensible, dentro de la férrea continuidad de la política comercial local (y también regional, a escala de Cataluña), a pesar de la rotación de los gobiernos socialista, nacionalista y populista.

Sin rehuir las polvaredas mediáticas o populares, desde el Observatorio del Comercio Urbano de la Universidad de Barcelona (OCUB) se trata de generar conocimiento en torno a este aspecto del siempre maltratado sector del comercio al por menor; esta tarea se realiza tanto a escala local y regional, como nacional e internacional ${ }^{4}$. El programa inicial fue formulado en el seminario de Rio de Janeiro, en junio de 2006.

Para ello en este artículo se aborda el ajuste del concepto de patrimonio a la actividad comercial, así como el impacto del éxito del turismo en las dinámicas económicas de la ciudad y de sus establecimientos comerciales, para a continuación tomar cuenta de la importancia del mercado inmobiliario en la localización de las actividades económicas. Una vez reafirmado el papel de actividad económica del comercio al por menor, se aborda el impacto de los diferentes tiempos de la ciudad, así como el papel de las apropiaciones y reapropiaciones sociales del espacio urbano en la vida de la ciudad. Ello permite avanzar unas primeras conclusiones hacia el futuro para abrir el debate académico.

3 El real decreto elaborado por el ministro Miguel Boyer (2/1985, de 30 de abril), fue completado por la Ley de Arrendamientos Urbanos de 1994 y sus consecuencias aclaradas por la sentencia del Tribunal supremo de 2013.

4 Los seminarios internacionales de Ciudad Comercio y Consumo se iniciaron en Rio de Janeiro (2006) y prosiguieron en Barcelona (2008), Sâo Paulo (2011), Nápoles (2013) y México D.F. (2015) (D’Alessandro, 2015, p. 9). El próximo se celebrará en Lisboa en 2017. 


\section{LA DIFÍCIL PATRIMONIALIZACIÓN DE LOS ESTABLECIMIENTOS COMERCIALES}

Existen, y se mezclan con asiduidad, muchos conceptos erróneos sobre las actividades y establecimientos de comercio al por menor. Se tiende a olvidar con frecuencia que se trata de una actividad económica que busca la minimización de los gastos y la maximización de los ingresos para mantener y aumentar, a ser posible, el beneficio. Se olvida que existen estrategias diversas para conseguir esta finalidad que permiten escoger diversas posiciones entre la competencia y la colaboración entre negocios diferentes. Se olvida que las actividades comerciales de productos básicos no constituyen un servicio público, sino que mayoritariamente, en la economía capitalista, buscan el equilibrio entre oferta y demanda en beneficio propio. Unos olvidan los valores sociales y culturales que entrañan los intercambios de bienes y de servicios, mientras otros pueden sobrevalorarlos olvidando su base netamente económica y materialista. La clasificación de los establecimientos comerciales deviene por ello más compleja que la de las actividades económicas desarrollada prolijamente por los diferentes servicios de estadística a nivel local, nacional o internacional ya que puede tenerse en cuenta, además del producto o servicio ofrecido, la superficie de venta, la localización absoluta o relativa, la frecuencia de uso o el tipo de relación jurídica entre el vendedor y sus suministradores, así como al público objetivo o segmento de demanda al que se destina principalmente. Como poco se atiende al peso del mercado inmobiliario en el gasto de la empresa de comercio al por menor.

Toda esa complejidad comúnmente ignorada total o parcialmente aumenta aún más en el comercio urbano. Las relaciones entre ciudad y actividades comerciales es antigua, duradera e intensa, situándose entre la simbiosis y el parasitismo. El libre mercado que regiría las actividades de intercambio deja de ser un mito abstracto para concretarse en unos agentes y unas estrategias determinadas, con características comunes a todas las ciudades y particulares en cada una de ellas. Dentro de esta temática, que exige de las Ciencias Sociales estudiosos rigurosos y profundos cuya ausencia y pobreza parece injustificable ${ }^{5}$, se inserta plenamente el papel simbólico y patrimonial que algunos establecimientos comerciales alcanzan a jugar en muchas ciudades.

El urbanista estadounidense Kevin Lynch (1918-1984) al diseñar su metodología para acercarse al conocimiento de la imagen de la ciudad estableció la necesidad de

5 En 1985, en conexión con el grupo de estudios del comercio de la Unión Geográfica Internacional que dirigía el profesor francés Alain Metton (n. en 1943), se iniciaron los trabajos para una definición de una nueva geografía comercial que tras investigaciones diversas se ha convertido en la reivindicación de una geografía del consumo (Carreras, 1989 y 2015). 
tener en cuenta los hitos urbanos ${ }^{6}$, puntos de referencia exteriores al observador, que podían ser de naturaleza muy diversa según cada persona (Lynch, 1960; pp. 75-78 en la traducción castellana). Los establecimientos comerciales de todo tipo son indudablemente hitos de referencia, para la situación y el conocimiento de la ciudad para muchos de sus ciudadanos y visitantes. Su apertura al espacio público de la calle, reforzada por el display comercial de escaparates, género, publicidad y anuncios les hace especialmente visibles. Las enseñas comerciales son tan antiguas casi como la actividad, ya que el comercio requiere su visualización rápida y eficaz en la atracción de compradores; desde las pinturas de las tiendas romanas, como las conservadas en Pompeya o los letreros medievales hasta el neón y los anuncios luminosos de la modernidad. Con ello, los establecimientos comerciales como hitos de referencia para los itinerarios cotidianos o esporádicos se convierten ellos mismos en ciudad, en cierta forma se patrimonializan, pasan a formar parte no solo de la ciudad, sino de la imagen que de la ciudad tienen sus ciudadanos y, por tanto, de su memoria; los ciudadanos y visitantes se apropian de la imagen de estos establecimientos, la internalizan y la usan como referencia para situarse dentro de la ciudad. Los establecimientos comerciales, enclaves privados en el espacio público, en las fachadas que delimitan calles y plazas de las ciudades, independientemente de sus dimensiones y de su apariencia, forman parte de la memoria colectiva de los ciudadanos e, incluso también de los visitantes; las guías turísticas, internacionales o locales contribuyen a una fijación selectiva en sus secciones de ocio y compras. Por ello cuando cierra un establecimiento comercial, un bar, un restaurante una tienda de confección desaparece lo que fuera un hito urbano para muchos, se vacía de alguna forma la memoria histórica, se pierde una referencia histórica de la vida cotidiana, incluso para quienes nunca han sido clientes de dicho establecimiento. De ahí la repercusión mediática de los cierres de determinados establecimientos.

De la misma forma que la urbanista francesa Françoise Choay (n. en 1925) puso de relieve que el interés por el patrimonio artístico y monumental aparece en los momentos de la destrucción asociada a los procesos de la revolución francesa (Choay, 1980), en los momentos de renovación y cambio urbano surgen las reivindicaciones conservacionistas respecto al patrimonio comercial urbano. En Barcelona, tras el éxito aparente de la celebración de los Juegos olímpicos de verano de 1992, se lanzó la gran transformación contemporánea que ha permitido hablar de un discutible y discutido modelo Barcelona (Bohigas, 1985, Busquets, 1992; Capel, 2005; Carreras, 2012). Todo empezó, como es lógico, en la ciudad histórica, administrativamente denominada ciudad vieja, fruto de uno de los dos ejes de la política urbana, la de la

6 Landmarks en el original, mojones en la traducción argentina de ediciones Infinito (Lynch, 1960) 
renovación del centro, junto con la monumentalización de la periferia. Esta última se centró esencialmente en el espacio público, mientras que la primera afectó de lleno los espacios privados, de uso residencial o comercial y de servicios. Así, desde principios de la década de 1990 se iniciaron los trabajos de elaboración de listados de establecimientos comerciales privados que pudieran tener algún interés especial por su antigüedad o por sus características artísticas exteriores o interiores.

El instituto municipal del paisaje urbano y la calidad de vida, gestor del catálogo patrimonial de la ciudad, tomó la iniciativa de la política de definir unos establecimientos que se llamaron emblemáticos (Ferrer, 2001) Desde el inicio de la campaña Guapos per sempre en 1994 y hasta 2007, año de la publicación de la segunda edición del libro conmemorativo del programa del ayuntamiento se otorgaron 125 placas a establecimientos que se consideraban de este tipo Todas las tiendas galardonadas cumplían los requisitos básicos: el primero y más importante, tener más de cincuenta años de antigüedad y haber mantenido con cuidado el carácter original de sus aspecto externo; en segundo lugar, seguir en el mismo emplazamiento y trabajar dentro de la misma rama del sector comercial. Unos años antes, en 2003, para la publicación del Atlas de Barcelona se identificaron ya un total de 86 establecimientos emblemáticos (Carreras, 2003), aproximadamente los mismos que formaban la lista de establecimientos la primera edición del libro Guapos per sempre (2004) y que únicamente repertoriaba aquellos que contaban más de 100 años. Las placas que se colocaban en el suelo, en la acera frente a la entrada del establecimiento, eran tan sólo un reconocimiento para apoyar al propietario en la conservación, pero no suponían ningún tipo de protección.

Desde entonces los censos sobre este tipo de establecimientos han ido cambiando y se han ido tornando quizás más exhaustivos, por cuyo motivo se han ido catalogando cada vez más establecimientos, aunque no se han colocado más placas. El último censo realizado por el ayuntamiento de Barcelona en 2012 identificó un total de 454 establecimientos, todos ellos situados en Ciutat Vella, Eixample, Sant Andreu, Gràcia, Sant Martí, Sarrià, Sants-Montjuic y les Corts, es decir la Barcelona histórica y en los centros de los antiguos municipios anexionados a la ciudad entre 1897 y 1902. Una lista mucho más larga que la de 2007 al ampliar los criterios de la catalogación, identificando 118 establecimientos "emblemáticos", y 336 establecimientos que forman parte de dos tipos de mención nuevos: 154 considerados como establecimientos "destacados" y el resto "mencionados", siendo los "destacados" los que cumplen un menor número de criterios para ser catalogados. El $92 \%$ de los establecimientos emblemáticos se localizan en los Distritos de Ciutat Vella y Eixample, mientras que respecto a los establecimientos destacados, la cifra se sitúa en un 
$77 \%$ en estos dos distritos. Con ello ha resultado que a medida que el criterio de catalogación era más laxo se ha ampliado su distribución territorial’.

Entre 1997 y 1999 el observatorio del comercio urbano de la universidad de Barcelona realizó un proyecto de investigación subvencionado por el Instituto del Paisaje Urbano, lo que permitió contrastar y debatir los criterios que deben utilizarse en la valoración de los elementos que pueden ser significativos en el paisaje de las ciudades, más allá de los de catalogación y conservación de monumentos. Se trató de realizar un primer censo de elementos del paisaje urbano de la ciudad de Barcelona en el que se combinaba el conocimiento y criterio de los estudiosos universitarios (geógrafos, historiadores, antropólogos, historiadores del arte) con la experiencia cotidiana de los ciudadanos convocados a través de un periódico local de gran difusión, El Periódico de Cataluña, y la cadena local de televisión BTV. Con ello se consiguió una primera recolección de elementos visuales que marcaban el paisaje de la ciudad en su conjunto (el característico color negro y amarillo de los taxis, o las baldosas del pavimento de las aceras) o de algunos lugares concretos regularmente distribuidos por todo el ámbito urbano (pequeñas capillas murales de santos, un termómetro luminoso comercial o algunos anuncios comerciales). No se planteaba tampoco ningún tipo de protección, pero se reconocía su valor colectivo y se animaba su conservación, además del registro de las imágenes e informaciones conexas (Carreras, 2001, pp. 19-20 y 49-50; AA.VV., 1999, pp. 175-176). Se promovió así una visión menos monumental del paisaje urbano, más allá de los estilos artísticos y de los autores consagrados, por lo tanto más democrática promoviendo la participación ciudadana y reconociendo los valores de la vida cotidiana.

Para el funcionamiento de los establecimientos comerciales, y para el conocimiento de la estructura comercial de una ciudad, es muy importante tener en cuenta, por un lado las dinámicas del mercado, en general, así como los cambios en el comportamiento y en los gustos de los consumidores. Los buenos comerciantes se ven obligados a estar siempre atentos a todo tipo de cambios, estar al día en las innovaciones de los productos y los servicios, sin olvidar incluso los aspectos formales y decorativos de sus establecimientos, y los publicitarios, lo que va en contra lógicamente de cualquier tipo de conservacionismo dificultando la patrimonialización. Por lo que respecta a la valoración específica de los establecimientos emblemáticos

7 La reacción de los responsables del ayuntamiento de Barcelona a la vista del mapa del atlas comercial fue muy airada, ya que consideraban que se olvidaba el interés del municipio por la periferia, donde se había invertido mucho. Pero el mapa sólo representa el resultado de una política de tipo arquitectónico que prima la antigüedad sobre cualquier otro criterio; no podían existir establecimientos de este tipo en barrios con menos de cincuenta años de historia y menor nivel socio-económico. Matar al mensajero, en este caso, a la fiel representación cartográfica de los datos no es coherente, y el mapa se publicó (Carreras, 2003; pp. 138-139). 
de Barcelona en la actualidad es importante analizar las variables que actúan de manera determinante en el devenir de estos establecimientos y que sitúan el debate sobre su futuro mucho más allá de lo puramente técnico y arquitectónico, así como de lo nostálgico y mediático. Los tiempos y ritmos de la arquitectura y del arte son siempre mucho más lentos que los de las actividades económicas y los de las modas y costumbres sociales y obedecen a causas totalmente distintas.

Para poder analizar adecuadamente el caso de Barcelona es necesario atender, por lo menos, dos aspectos distintos y complementarios. Por un lado, los efectos del comportamiento del turismo de compras y consumo en la ciudad. Por otro lado, las consecuencias de la evolución del mercado inmobiliario en lo que respecta al aumento del valor de la propiedad del suelo y de los locales y de los alquileres.

\section{EL TURISMO COMO FACTOR MULTIPLICADOR DEL CONSUMO Y FAVORECEDOR DE LA PATRIMONIALIZACIÓN}

Barcelona es hoy sin duda la principal ciudad turística de España con alrededor de 8 millones de turistas y visitantes al año (Ajuntament de Barcelona, 2014) y la cuarta de Europa, después de Londres, París e Estambul, y la duodécima del mundo, en un ranquin más difícil y discutible probablemente. El $50 \%$ de los turistas que visitan la ciudad lo hacen con motivo de vacaciones, el $40 \%$ por negocios y un $10 \%$ por ferias y congresos.

En lo que se refiere al consumo, según Mastercard Global Destination Cities Index 2014, Barcelona fue la séptima ciudad con mayor gasto de turistas internacionales del mundo ${ }^{8}$. El mismo organismo establece que la ciudad de Barcelona es la segunda ciudad del mundo con gasto de turista internacional por residente en la ciudad, con 2.793 euros por habitante. ${ }^{9}$ Este hecho hace que a pesar de lo corto de las estancias individuales en la ciudad, los turistas se convierten en un grupo consumidor destacado y permanente a lo largo del año en la ciudad (Carreras et al, en prensa). A pesar de la diversidad de perfiles socioeconómicos y culturales de los turistas barceloneses, se detectan unos patrones de consumo más semejantes entre ellos que si la variable consideraba fuera la edad, por ejemplo.

A partir de los datos que ofrecen las empresas propietarias de las tarjetas de crédito que han sido tratados por Turisme de Barcelona (2014), se cree que el 33\% del

8 Por detrás de Londres, Nueva York, París, Singapur, Bangkok, Seúl y por delante de Dubai, Taipéi y Estambul.

9 En primer lugar se sitúa Dubai con 4.668 euros de gasto por turista internacional y habitante residente. Estas cifras se explican sobre todo por la reducida superficie municipal y, por tanto, de habitantes de la ciudad respecto a su área metropolitana. 
gasto turístico por persona se realiza en establecimientos comerciales, un porcentaje que representaría un promedio de 64 euros diarios. ${ }^{10}$ Evidentemente no todos los turistas gastan de igual forma, en el caso de Barcelona, chinos y rusos, eran las nacionalidades con mayor gasto en la ciudad; de igual forma, a pesar de que el perfil mayoritario del turista en Barcelona es el de personas de entre 25 y 34 años, representando un $34 \%$ del total, los turistas de mayor edad consumen más en los establecimientos comerciales que los más jóvenes.

Estas cifras no son nada despreciables para ayudar a entender las transformaciones que han experimentado las actividades comerciales de Barcelona, en su conjunto, y en algunos sectores centrales en particular. El gasto diario por persona de los turistas en establecimientos comerciales es muy superior al de los residentes en la ciudad lógicamente, por cuyo motivo de manera creciente los establecimientos comerciales se han ido enfocando cada vez más hacia los turistas ${ }^{11}$. El proceso de modernización de los productos y servicios, de las técnicas de venta y del display comercial ha sido más visible especialmente durante estos últimos años de crisis social y económica que ha afectado de manera directa a la capacidad de consumo de los ciudadanos barceloneses y españoles en general. A pesar de que ha habido adaptaciones específicas de los establecimientos comerciales a los requerimientos de consumo de los turistas, tal y como ya se demostró analizado la estructura comercial del barrio del Raval (Carreras et al, en prensa), de manera general la presencia tan importante de turistas en la ciudad durante todo el año y la difusión creciente de los lugares consumidos por los visitantes, hace pensar que de manera general, salvo en los barrios más periféricos, toda la estructura comercial de Barcelona se ha visto beneficiada por los ingresos generados por el turismo.

La adaptación de algunos establecimientos emblemáticos de Barcelona al turista consumidor ha actuado también como elemento conservador e incluso dinamizador y no como en algunos medios se difunde, debilitando estos establecimientos o acabando con ellos. En algunas ocasiones se ha podido determinar cómo se ha gentrificado a algunos establecimientos comerciales tradicionales, convirtiendo la antigüedad en una pátina atractiva (Martínez-Rigol, Moreno, 2008). Muchos de los establecimientos emblemáticos más conocidos se han convertido en atractivo turístico, a partir de su

10 Los restantes euros hasta los $189 €$ totales diarios gastados por turista (la cifra más alta de España según la "Encuesta de Gasto Turistica" del INE) forman parte del alojamiento, transporte, comida y principalmente entradas a los monumentos. El gasto total de los turistas internacionales que visitaron Barcelona durante el mayo de 2016 alcanzó la suma de 1.541 millones de euros, durante los primeros cinco meses del año la cifra alcanzó los 5.238 millones de euros. Si se mira el gasto diario medio por turista la cifra asciende a esos 189 euros diarios comentados.

11 El primer gran estudio comercial de la ciudad de Barcelona de la primera mitad de los años 1980 se subtitulo de forma reivindicativa con el eslogan: Barcelona ciudad de compras (Carreras, Domingo, Sauer, 1990). 
difusión en las guías y artículos de periódicos y revistas de todo tipo. Ello es quizás especialmente claro en el caso de algunos restaurantes, como el caso que se ejemplifica en la figura 1.

Figura 1 - Portada de la guía turística de Barcelona editada por Lonely Planet (2010) con la imagen de la entrada del restaurante Los caracoles, establecimiento emblemático de Ciutat Vella.

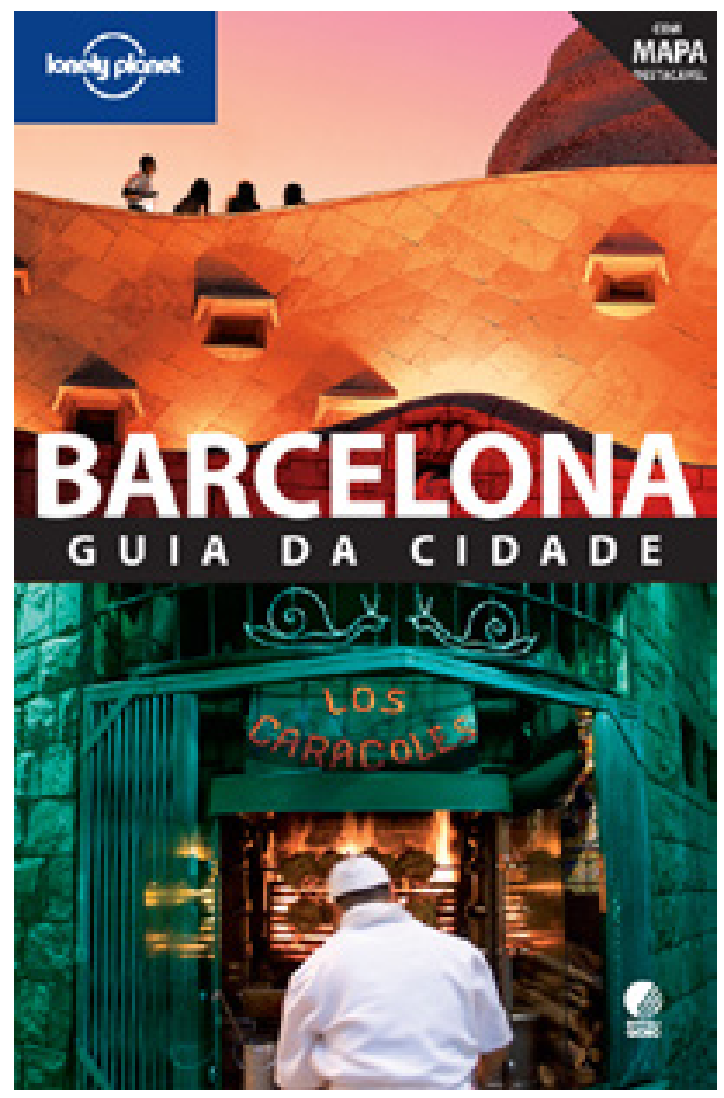

\section{EL IMPACTO DEL MERCADO INMOBILIARIO EN LOS ESTABLECI- MIENTOS COMERCIALES}

El mercado inmobiliario ejerce un papel trascendental en la configuración, evolución y transformaciones de la ciudad y, por tanto, de la estructura comercial. Los locales comerciales, como cualquier otro solar o local destinado a alguna función urbana, como por ejemplo la residencial, probablemente mucho más conocida, han experimentado un creciente proceso de mercantilización y de globalización (Bernardos,et al, 2014). Sin lugar a dudas, la mercantilización de los locales comerciales suele tener unos efectos mucho menos dramáticos si se compara con los del 
mercado de la vivienda, dónde a pesar de existir una mayor oferta, el acceso a ella por una parte muy importante de la demanda es muy difícil, cuando no imposible.

El precio de los locales comerciales, lógicamente, es producto de la relación entre oferta y demanda que en el caso del mercado inmobiliario, depende en una gran parte de su localización, tanto absoluta, dentro del espacio urbano, como relativa, respecto a otras localizaciones similares o competitivas. Este hecho explica la formación de la renta diferencial del suelo que tiene un efecto directo en el precio final del local. La formación de la renta diferencial del suelo permitió en la década de los años 1960 la formulación del modelo sobre usos del suelo y localización del economista argentino William Alonso (1933-1999). Este modelo, de acuerdo con su simplificación neopositivista y en una concepción isotrópica del espacio urbano, establecía que las actividades con capacidad para pagar las rentas del suelo más altas, dada su necesidad de estar en el lugar más central y frecuentado eran las comerciales.

El papel del mercado inmobiliario como motor para entender las transformaciones de Barcelona se inició a partir de la segunda mitad del siglo XIX, con la apertura de las tierras del llano circundante a su plena urbanización, a través de la aprobación del plan Cerdà, en 1860 (Tafunell, 1989). Aunque no ha sido aun suficientemente investigada, la internacionalización del mercado inmobiliario barcelonés se produjo a partir de la integración de España en la UE en 1986, más aún a partir de la entrada en la moneda única en 2001 que acabó con el diferencial de divisa (Bernardos et al, 2014). El éxito turístico posterior a Barcelona 1992 y el boom inmobiliario de finales de milenio multiplicaron la demanda de propiedades urbanas. Grandes compañías inmobiliarias internacionales empezaron a operar en el mercado residencial, pero también en otros sectores con suficiente demanda, como el de las naves logísticas y las oficinas, profundamente afectados por la crisis iniciada en 2007, así como el de locales comerciales, también afectado por la depresión pero menos, sobre todo en el caso de las localizaciones más centrales de la ciudad.

El aumento del precio de los locales comerciales como resultado del incremento del peso del mercado inmobiliario no ha dejado de crecer, especialmente en el centro de la ciudad de Barcelona (Figura 2) a pesar de la crisis social y económica de 2007. Como puede apreciarse, la evolución ha sido más positiva en las simbólicas calles de Portal del Ángel y paseo de GraciaEn algunas ocasiones la revalorización de las rentas del suelo ha sido tan elevada que algunas empresas comerciales han pasado a comprar los inmuebles para, posteriormente alquilarlos a otras cadenas comerciales o ubicar una tienda de su cadena, tal como ha pasado con el gigante de 
la moda INDITEX, en sus dos inmuebles de propiedad de la plaza de Cataluña dónde e se ubica un establecimiento de Zara o el de la cadena Apple.

Figura 2 - Evolución de los precios en $€$ por $\mathrm{m}^{2}$ de alquiler de local comercial en diversas calles del centro de Barcelona

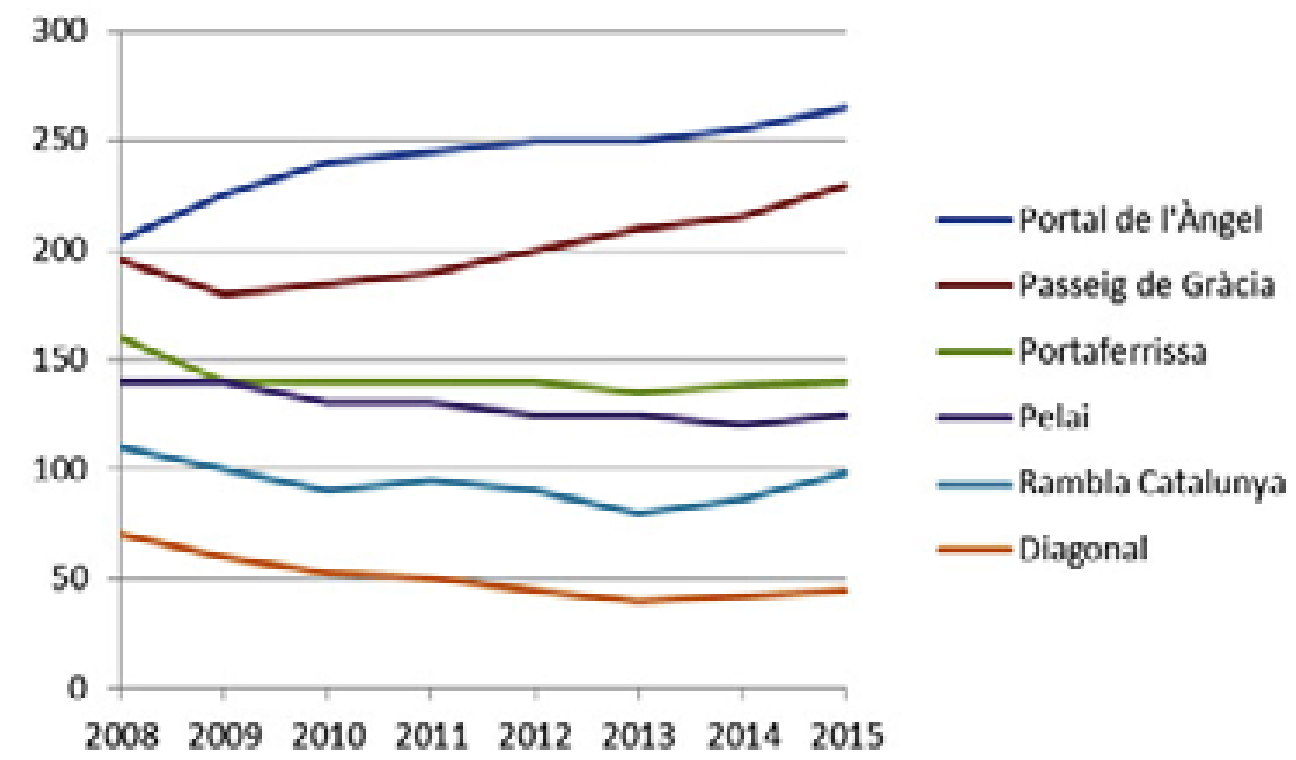

El peso del mercado inmobiliario ha tenido sus efectos en toda la estructura comercial de Barcelona, especialmente en los establecimientos emblemáticos dada la antigüedad de sus contratos y alquileres y su localización mayoritariamente central. Lógicamente los efectos sobre los negocios comerciales han sido muy distintos según el régimen de tenencia del local comercial. Los establecimientos, emblemáticos o no, en régimen de alquiler con contratos anteriores a 9 de mayo de 1985, cuando fue aprobado el famoso decreto Boyer, se regían por el decreto de arrendamientos urbanos 4104/1964, de 24 de setiembre, quedando sujetos, con carácter imperativo, al régimen de prórroga forzosa durante 20 años más. Más tarde, la Ley 29/1994, de 24 de noviembre, de arrendamientos urbanos, vigente desde el 1 de enero de 1995, eliminó toda exigencia forzosa de duración o prórroga obligatoria de los contratos de arrendamiento para uso distinto a los de la vivienda. De esta manera, la ley preveía la extinción de los contratos de renta antigua, y su consecuente actualización a las reglas del mercado actuales, tanto por lo que respecta a los valores del alquiler como en lo referente a la duración del contrato.

En abril de 2012, el ayuntamiento de Barcelona realizó una encuesta a los es- 
tablecimientos "emblemáticos" y "destacados" constatando que en el $60 \%$ de los casos el régimen de tenencia era de alquiler. Dentro de este grupo, también un $60 \%$ de los negocios se regían por las rentas antiguas, significando un 31\% de los 272 establecimientos censados como "emblemáticos" o "destacados". Estos establecimientos estaban así afectados por la caducidad que establecía la disposición transitoria tercera de la LAU (Llei 29/1994). Estos mismo datos revelan que en el $40 \%$ de los casos, el empresario-comerciante de los establecimientos emblemáticos y destacados también es propietario del local comercial, con lo cual el problema no existe; tan sólo se le presenta la posibilidad de hacer un buen negocio inmobiliario, en coherencia con la fase de capitalismo financiero que ha sucedido al comercial y al industrial.

La problemática, por tanto, es distinta para el caso de los establecimientos abiertos en locales de alquiler, de los que lo están en locales de propiedad. En los primeros resulta difícil poder explicar con certeza cuando el cierre del negocio se debe exclusivamente al aumento del precio del alquiler o a la disminución de la facturación y de los beneficios. De una forma parecida, pero con consecuencias muy diferentes, en los establecimientos comerciales en locales de propiedad resulta difícil saber con precisión cuando la venta se produce por motivos puramente inmobiliarios o cuando éstos vienen justificados por una disminución del beneficio o por pérdida de interés en la continuidad del negocio. Ante el cierre del establecimiento Vinçon, en el paseo de Gracia de Barcelona, dedicado a la venta de productos de diseño, el propietario del negocio señalaba significativamente en este sentido que la única solidaridad que podía salvar el negocio era recuperar el número de clientes.

Catálogos y encuestas de las administraciones, libros periodísticos bellamente ilustrados e intervenciones mediáticas lanzando protestas jeremíacas no han conseguido ni analizar, ni ayudar a resolver el problema. Des 1985, el ayuntamiento de Barcelona ha tenido tres administraciones políticas distintas y en ningún caso se ha previsto el problema ni se han avanzado medidas efectivas de ayuda a la actividad comercial que da origen a los establecimientos (a los emblemáticos, a los destacados y a los que no son considerados ni lo uno, ni lo otro). La liberación de los precios y alquileres se ha tratado como una catástrofe natural inevitable y muy pocos han cuestionado el sistema liberal que subyace al funcionamiento del mercado inmobiliario. El establecimiento de alguna tasa o impuesto municipal al cambio de actividad podría tal vez haber constituido una acción práctica.

Así, el precio del suelo y de los locales continua siendo el motor de la producción del espacio urbano, cuya lógica se impone por encima de las lógicas de los negocios 
y de las actividades privadas. A pesar de las estrechas relaciones que existen entre el comercio al por menor y la vida urbana, los establecimientos comerciales son negocios privados y no un servicio público, ni siquiera aquellos que, como las farmacias o las panaderías, se habían organizado en Barcelona de una forma gremial y territorialmente organizada.

\section{LA EVOLUCIÓN RECIENTE DE LOS ESTABLECIMIENTOS EMBLEMÁ- TICOS BARCELONESES}

Entre los años 1994 y 2007 se habían otorgado un total de 125 placas conmemorativas a otros tantos establecimientos; primero a los que tenían en torno a cien años, posteriormente a los que rondaban los 50. En 2007, ya se habían retirado 6 placas por traspaso o cambio del negocio. En cambio, durante los últimos 10 años han cerrado 36 establecimientos más de los considerados emblemáticos, restando abiertos actualmente tan sólo 83, es decir la dos terceras partes de la cifra total (Figura 3). Este aumento del cierre de establecimientos coincide con la comentada liberalización de alquileres, pero también con las consecuencias de la crisis del 2008. Pero no por ello el ruido mediático ha sido menor.

Desde un punto de vista territorial, la concentración de establecimientos abiertos y sobre todo cerrados es mucho mayor en las calles de la ciudad histórica, y, aunque algo menos en el sector central del Ensanche del siglo XIX. Fuera de estos ámbitos la presencia de establecimientos repertoriados es prácticamente simbólica, a causa de la visión historicista y arquitectónica aplicada. Igualmente se aprecia como la calles que experimentan los mayores precios del suelo no albergan ninguna cantidad significativa de este tipo de establecimientos comerciales que obedeces a lógicas muy diferentes de las del mercado inmobiliario.

Los establecimientos que mejor han resistido los cambios y las crisis son los dedicados a la farmacia, a la restauración y a la hostelería; se trata de negocios que mantienen una demanda constante sino en aumento, especialmente los últimos empujados por el comentado auge del turismo. Pero aun manteniéndose formalmente, algunos establecimientos han cambiado totalmente su actividad comercial. Tal es el caso de la pastelería Escribà, en plenas Ramblas barcelonesas que desde el año 1986, seis años antes tan sólo del inicio de la política conservacionista, adquirió el local de la antigua fábrica de pastas de sopa Figueras, instalada en 1842 y reformada en estilo art nouveau en 1902. Como la joyería Roca del paseo de Gracia, de estilo racionalista del famoso arquitecto Josep Lluís Sert (1902-1983), que se ha convertido en un establecimiento de la cadena de joyerías Tous. Como la pastelería 
Reñé, de la calle del Consell de Cent, fundada en 1910 y que desde el año 2000 se ha convertido en un restaurante que conserva la fachada y algunos elementos de la decoración anterior. La política en estos casos protege claramente la arquitectura pero no la actividad comercial.

Figura 3 Localización de los establecimientos emblemáticos abiertos y cerrados y de las calles comerciales más valoradas en la figura 2.

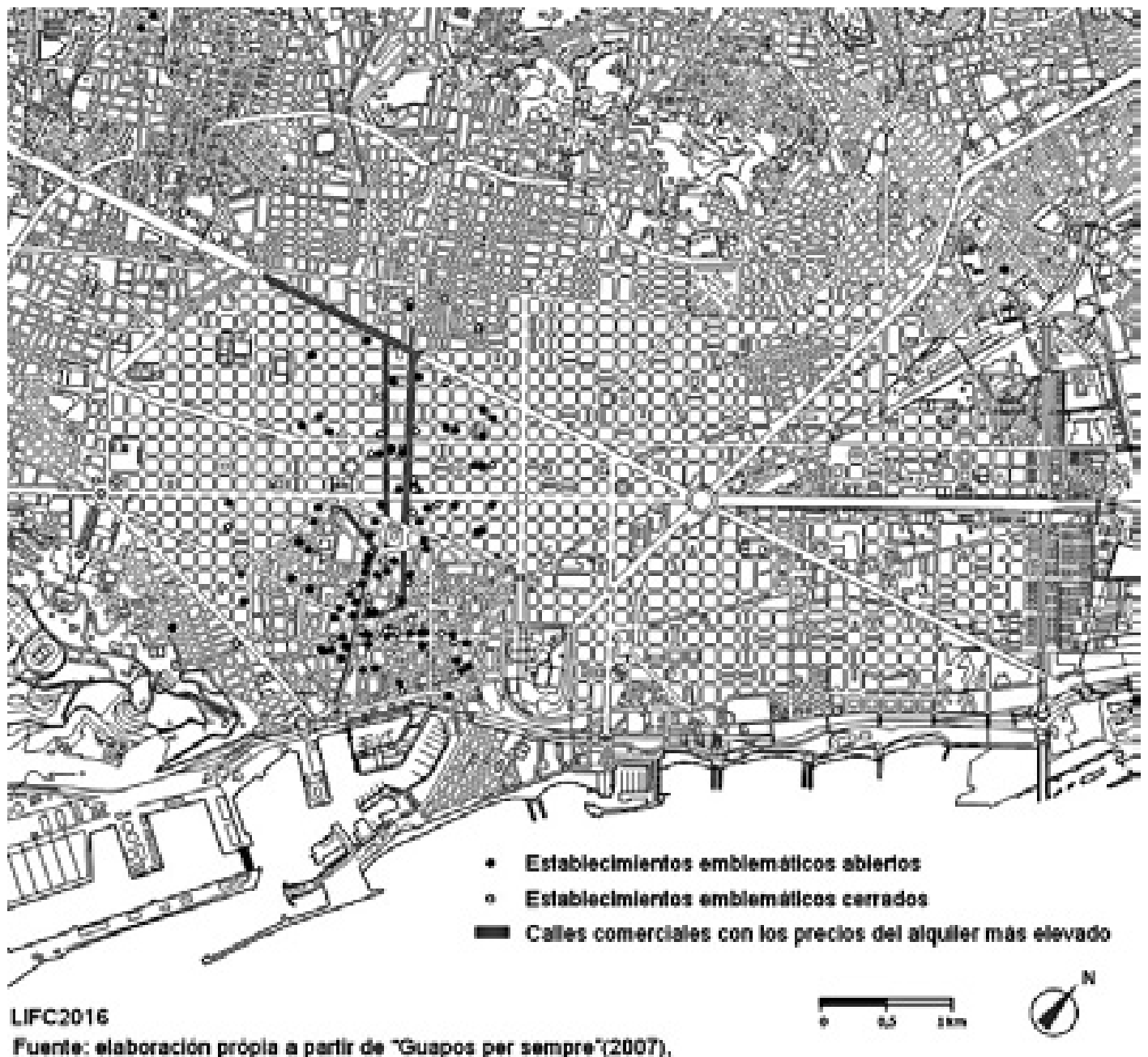
trabajo de campo y base cartografica OCUB.

\section{CONCLUSIONES DE FUTURO}

A partir del caso de la ciudad de Barcelona, queda plenamente acreditado el carácter patrimonial e identitario, de referencia urbana en la vida cotidiana de muchos 
establecimientos comerciales y de servicios12. El éxito del consumo turístico aparece como un factor favorable a la conservación de los rasgos identificadores de una ciudad, de un barrio o de una calle, al menos en sus aspectos formales.

El primer problema a plantear es el de una definición adecuada y completa de lo que debe ser considerado un establecimiento emblemático. Un único criterio casi exclusivamente material, arquitectónico presenta limitaciones. En una investigación sobre el patrimonio comercial de Cataluña que quería destacar su papel en la memoria de la vida cotidiana, se avanzaron tres criterios diferentes que no siempre se daban en su totalidad. Un primer criterio, efectivamente, puede ser el de la calidad y originalidad del edificio en el que se encuentra localizado el establecimiento; un segundo criterio, más relacionado ya con la actividad comercial era la calidad artística de la decoración, publicidad y display del establecimiento; un tercer criterio, plenamente comercial es la calidad y originalidad de los productos o servicios que se ofrecen (Carreras, Moreno y Ariño, 2006).

A esos criterios debe añadirse aún la variable temporal que rehúya establecer como preferente o normativo un estilo o un momento sobre otros; el carácter dinámico de las actividades comerciales y del consumo obliga a tener en cuenta la diversidad de gustos y de momentos, dando cabida no sólo a la tradición, sino también a la modernidad y a la innovación. Ello debido a las difíciles relaciones entre los distintos ritmos temporales que se dan en la ciudad y en las apropiaciones diferentes del paisaje urbano por parte de los ciudadanos y de los visitantes. Especialmente compleja es la relación entre lo permanente de lo urbano y lo efímero de las modas que eclosiona en todos y cada uno de los establecimientos comerciales.

Las reacciones mediáticas no suelen ayudar a la comprensión de los problemas que genera la complejidad, sino que acostumbran a responder a intereses puntuales e inmediatos y pasajeros, a menudo más efímeros que las modas. Dichas reacciones en Barcelona se han dado apelando a la nostalgia, frente a la lógica económica de las empresas comerciales, y enfrentando las poblaciones locales, los llamados vecinos, a los ciudadanos foráneos, los turistas, esgrimiendo la masificación como argumento. Muchas veces se encuentra en la base de las campañas de los medios de comunicación el conflicto permanente entre el nosotros, más o menos identitario, y el ellos, el otro, a veces turista, a veces inmigrante; problemática muy alejada de la que se refiere a los establecimientos comerciales emblemáticos y a su supervivencia en la ciudad. Como se ha visto, a través de las guías e itinerarios turísticos

12 También los edificios públicos (escuelas, hospitales, administración) y religiosos tienen un papel similar, pero su conservación es competencia exclusiva de sus administraciones, lo que no obsta para su demolición o renovación con mayores consecuencias negativas para el imaginario colectivo ciudadano. 
el conservacionismo de los establecimientos emblemáticos se ve más favorecido que perjudicado; ello se puede corroborar en las apropiaciones cotidianas que los diversos grupos de turistas realizan de partes de la ciudad, especialmente de los sectores centrales.

Una conclusión más general respecto al caso analizado y con aplicaciones posibles a muchos otros casos es la necesidad de superar el tratamiento del problema a nivel de establecimiento individual. Las actividades comerciales han tejido desde el origen de su historia densas interrelaciones entre especialidades y modalidades que se han concretado en la formación de las ferias y los mercados, de las calles y plazas mayores, de los centros y áreas comerciales. En este sentido, parece necesario que las administraciones locales se planteen el paso de superar la consideración del establecimiento emblemático hacia el planteamiento de la urbanización, apoyo y conservación de la calle o plaza comercial emblemática. Con ello la simbiosis entre comercio al por menor y ciudad y vida urbana alcanzaría un nivel más adecuado de consideración social y política.

\section{BIBLIOGRAFÍA CITADA}

AA.VV. (1999), Barcelona, posa't guapa. Tretze anys.Barcelona: Ajuntament de Barcelona; 296 pp.

Ajuntament de Barcelona (2014), El comerç a Barcelona. Ajuntament de Barcelona. P.175 Ajuntament de Barcelona (2015), Catàleg de patrimoni arquitectònic, històric-artístic i paisatjístic dels establiments emblemátics de La ciutat de Barcelona. Ajuntament de Barcelona, 2016.

Ascana, (2008-2016), Informe locales comerciales. Madrid: Ascana Expansión.

Bernardos, G., Martínez-Rigol, S., Frago, L. y Carreras, C. (2014), “Las apropiaciones de la ciudad a la hora de la globalización: las estrategias del capital ruso y chino en el mercado inmobiliario de Barcelona" en Scripta Nova, Barcelona: Universitat de Barcelona; vol. 18, no 493(52), pp. 1-17

Capel, H. (2005) El modelo Barcelona, un examen crítico. Barcelona: Ediciones El Serbal. Carreras, C., (1989), "Per una nova geografía comercial", en Revista Catalana de Geografia, Barcelona: ICC; vol IV, no 10; pp. 5-16.

Carreras, C. (2001), La universitat i la ciutat. Barcecelona: Aula Barcelona; 68 pp.

Carreras, C. ( 2003) Atles comercial de Barcelona. Barcelona: Ajuntament de Barcelona. 
Carreras, C. (2012) "De modelos urbanos: una revisión histórica a partir del caso de Barcelona" en Boletín de la Real Sociedad Geogràfica, Madrid; vol. CXLVIII; pp. 183-204

Carreras, C. (2015), “For a more critical Consumption history” in D’Alessandro, L. (2015).

Carreras, C., Domingo, J. y Sauer, C. (1990), Les àrees de concentració comercial de Barcelona. Barcelona ciutat de compres. Barcelona: Institut Cartogràfic de Catalunya.

Carreras, Martinez, Frago, Morcuende, Montesinos,...(en prensa)

Carreras, Moreno, Ariño, 2006, Las tiendas históricas de Cataluña,

Choay, F. (1980), La Règle et le Modèle : Sur la théorie de l'architecture et de l'urbanisme, Paris, Seuil,

D'Alessandro, L. (2015), City, Retail and Consumption. Napoli: Università degli Studi di Napoli "L’Orientale"; 478 pp.

Ferrer, F. (2001), El Paisatge urbà, un punt de trobada. Barcelona : Universitat de Barcelona; $95 \mathrm{pp}$.

Frago, L. (2011) La metamorfosi del centre a les capitals comarcals: entre tradició i postmodernitat. Tesis doctoral. Barcelona: Universitat de Barcelona.

Hedrick-Wong, Y. \& Choong, D. (2015), MasterCard Global destination cities Index. New York: MasterCard.

Lynch, K. (1960) The Image of the City. Cambridge, Mass.: The Massachusset Institute of Technology Press [traducción castellana, Buenos Aires: ediciones Infinito, 1974, 3a edición]

Martinez-Rigol, S. y Moreno, S. (2008)

Moreno, S. (2011) Aproximación teórica y práctica a las relaciones entre comercio y ciudad. El caso de laproducción y el consumo de libros en la ciudad de Barcelona. Tesis doctoral. Barcelona: Universitat de Barcelona.

Tafunell, X. (1989), La construcción residencial en el crecimiento residencial de Barcelona (1854-1897). Barcelona: Universitat Autònoma de Barcelona; Tesis doctoral. 